\title{
Revisiting Riesz transforms on Heisenberg groups
}

\author{
P. K. Sanjay and Sundaram Thangavelu
}

Dedicated to Eli Stein on his eightieth birthday.

\begin{abstract}
We characterise higher order Riesz transforms on the Heisenberg group and also show that they satisfy dimension-free bounds under some assumptions on the multipliers. Using transference theorems, we deduce boundedness theorems for Riesz transforms on the reduced Heisenberg group and hence also for the Riesz transforms associated to multiple Hermite and Laguerre expansions.
\end{abstract}

\section{Introduction}

The aim of this paper is twofold: to prove dimension-free estimates for Riesz transforms associated to reduced Heisenberg groups $\mathbb{H}_{\text {red }}^{n}$ and to study Riesz transforms of higher order on Heisenberg groups $\mathbb{H}^{n}$ which are in a sense canonical. Dimensionfree estimates for Riesz transforms on $\mathbb{H}^{n}$ have been studied in the literature by Coulhon et al. [3] but the proof given there, which depends very much on the dilation structure of $\mathbb{H}^{n}$, does not work for the reduced Heisenberg group. However, we can view the Riesz transforms on the Heisenberg group (reduced Heisenberg group) as an operator valued multiplier for the Fourier transform (resp. Fourier series). Hence using a transference theorem we can deduce results for the reduced Heisenberg group from those for $\mathbb{H}^{n}$. Let us set up the notation before stating our results.

Let $\mathbb{H}^{n}=\mathbb{C}^{n} \times \mathbb{R}$ denote the $(2 n+1)$-dimensional Heisenberg group with the group operation $(z, t)(w, s)=\left(z+w, t+s+\frac{1}{2} \operatorname{Im}(z \cdot \bar{w})\right)$. Its Lie algebra $\mathfrak{h}_{n}$ is generated by the $(2 n+1)$ left invariant vector fields

$$
T=\frac{\partial}{\partial t}, \quad X_{j}=\left(\frac{\partial}{\partial x_{j}}+\frac{1}{2} y_{j} \frac{\partial}{\partial t}\right), \quad Y_{j}=\left(\frac{\partial}{\partial y_{j}}-\frac{1}{2} x_{j} \frac{\partial}{\partial t}\right), \quad j=1,2, \ldots n .
$$

Mathematics Subject Classification (2010): 47B35, 43A85, 22E30.

Keywords: Riesz transforms, Heisenberg groups, Fourier multipliers, Hermite functions, Laguerre functions. 
The operator $\mathcal{L}=-\sum_{j=1}^{n}\left(X_{j}^{2}+Y_{j}^{2}\right)$ is called the sublaplacian on $\mathbb{H}^{n}$. Written explicitly,

$$
\mathcal{L}=-\Delta_{z}-\frac{1}{4}|z|^{2} \frac{\partial^{2}}{\partial t^{2}}+N \frac{\partial}{\partial t},
$$

where $\Delta_{z}$ is the ordinary Laplacian on $\mathbb{C}^{n}$ and

$$
N=\sum_{j=1}^{n}\left(x_{i} \frac{\partial}{\partial y_{j}}-y_{i} \frac{\partial}{\partial x_{j}}\right)
$$

We can also write $\mathcal{L}$ as

$$
\mathcal{L}=-\frac{1}{2} \sum_{j=1}^{n}\left(Z_{j} \bar{Z}_{j}+\bar{Z}_{j} Z_{j}\right)
$$

where $Z_{j}=\left(X_{j}-i Y_{j}\right)$ and $\bar{Z}_{j}=\left(X_{j}+i Y_{j}\right)$ for $j=1,2, \ldots n$. We define the Riesz transforms

$$
R_{j}=Z_{j} \mathcal{L}^{-1 / 2} \quad \text { and } \quad \bar{R}_{j}=\bar{Z}_{j} \mathcal{L}^{-1 / 2}, \quad j=1,2, \ldots, n
$$

associated with $\mathcal{L}$.

These operators are known to be singular integral operators on $\mathbb{H}^{n}$ and hence bounded on $L^{p}\left(\mathbb{H}^{n}\right), 1<p<\infty$, and of weak type $(1,1)$. Moreover, in [3] it is proved that the bounds do not depend on the dimension of $\mathbb{H}^{n}$. In this work we consider the same operators acting on the reduced Heisenberg group $\mathbb{H}_{\text {red }}^{n}=\mathbb{H}^{n} / \Gamma$, where $\Gamma=\{(0,2 \pi k): k \in \mathbb{Z}\}$ is a central subgroup (see Chapter 4 of [22] for details). Thus functions on $\mathbb{H}^{n} / \Gamma$ are $2 \pi$ periodic in the central variable. For the Riesz transforms acting on $L^{p}\left(\mathbb{H}^{n} / \Gamma\right)$ we prove the following theorem:

Theorem 1.1. For each $1<p<\infty$, there exists a constant $C_{p}$, independent of the dimension $n$, such that for all $f \in L^{p}\left(\mathbb{H}^{n} / \Gamma\right)$,

$$
\left\|\left(\sum_{j=1}^{n}\left|R_{j} f\right|^{2}+\sum_{j=1}^{n}\left|\bar{R}_{j} f\right|^{2}\right)^{1 / 2}\right\|_{p} \leq C_{p}\|f\|_{p} .
$$

As we mentioned earlier, we deduce this theorem from the result of [3] on the Heisenberg group. The appropriate transference theorem is stated in Section 2.

We now turn our attention to higher order Riesz transforms on the Heisenberg group. Observe that the Riesz transforms defined as $\left(X_{j}-i Y_{j}\right) \mathcal{L}^{-1 / 2}$ and $\left(X_{j}+i Y_{j}\right) \mathcal{L}^{-1 / 2}$ are multipliers for the group Fourier transform. The corresponding multipliers are given by $A_{j}(\lambda) H(\lambda)^{-1 / 2}$ and $A_{j}^{*}(\lambda) H(\lambda)^{-1 / 2}$ respectively, where for $j=1,2, \ldots, n$ and $\lambda \in \mathbb{R}, A_{j}(\lambda)$ and $A_{j}^{*}(\lambda)$ are the creation and annihilation operators:

$$
A_{j}(\lambda)=-\frac{\partial}{\partial \xi_{j}}+\lambda \xi_{j}, \quad A_{j}^{*}(\lambda)=\frac{\partial}{\partial \xi_{j}}+\lambda \xi_{j}
$$

and

$$
H(\lambda)=-\Delta+\lambda^{2}|x|^{2}=\frac{1}{2} \sum_{j=1}^{n}\left(A_{j}(\lambda) A_{j}^{*}(\lambda)+A_{j}^{*}(\lambda) A_{j}(\lambda)\right)
$$


is the Hermite operator. We would like to consider higher order Riesz transforms on $\mathbb{H}^{n}$ as Fourier multipliers corresponding to higher order analogues of $A_{j}(\lambda) H(\lambda)^{-1 / 2}$ and $A_{j}^{*}(\lambda) H(\lambda)^{-1 / 2}$. In analogy with the case of the standard Laplacian on $\mathbb{R}^{n}$, the above operators can be considered as analogues of the Riesz transform for the Hermite expansions. Moreover, as it was shown in [21], they turn out to be pseudodifferential operators of order zero and hence bounded on $L^{p}\left(\mathbb{R}^{n}\right), 1<p<\infty$.

Higher order Riesz transforms associated to the Hermite operator have also been studied in the literature by defining them as operators of the form $A(\lambda)^{\alpha}$ $H(\lambda)^{-\frac{1}{2}|\alpha|}$ and $A^{*}(\lambda)^{\alpha} H(\lambda)^{-\frac{1}{2}|\alpha|}$, where $\alpha$ is a multiindex and $A(\lambda)=\left(A_{j}(\lambda)\right)$, $A^{*}(\lambda)=\left(A_{j}^{*}(\lambda)\right)$ are vectors, see [8] and [12]. Here we consider the operators $G_{\lambda}(P) H(\lambda)^{-\frac{m}{2}}$ as the natural candidates for the higher order Riesz transforms, where $P$ is a bigraded solid harmonic on $\mathbb{C}^{n}$ and $G_{\lambda}(P)$ is the operator associated to $P$ by the Weyl correspondence. (For the definition of $G_{\lambda}(P)$, as well as the Weyl transform $W_{\lambda}(P)$, we refer to Section 3; more details can be found in [23].) This class of operators includes the preceding ones, because $P(z)=z^{\alpha}$ and $Q(z)=\bar{z}^{\alpha}$ are bigraded solid harmonics of bidegrees $(|\alpha|, 0)$ and $(0,|\alpha|)$, and by a theorem of Geller [6], $G_{\lambda}(P)=A(\lambda)^{\alpha}$ and $G_{\lambda}(Q)=A^{*}(\lambda)^{\alpha}$.

Theorem 1.2. For every bigraded solid harmonic $P$ of total degree $m$, the operator $G_{\lambda}(P) H(\lambda)^{-\frac{m}{2}}$ is bounded on $L^{p}\left(\mathbb{R}^{n}\right), 1<p<\infty$, and is of weak type $(1,1)$.

This theorem can be deduced, in principle, from the known results on higher order Riesz transforms. However, we prefer to give a painless proof which is based on Mauceri's transference theorem [13] for Weyl multipliers. In view of this transference, we only need to show that $G_{\lambda}(P) H(\lambda)^{-\frac{m}{2}}$ is an $L^{p}$ multiplier for the Weyl transform $W_{\lambda}$. The operator $T_{P}$ defined on $L^{2}\left(\mathbb{C}^{n}\right)$ by

$$
W_{\lambda}\left(T_{P} f\right)=W_{\lambda}(f) G_{\lambda}(P) H(\lambda)^{-\frac{m}{2}}
$$

turns out to be a twisted convolution operator with a singular kernel. Thanks to the Hecke-Bochner type formula for the Weyl transform, due to Geller [6], the kernel can be estimated easily. By appealing to the theory of oscillatory singular integrals developed by Ricci and Stein [16] and Chanillo and Christ [1] we can prove

Theorem 1.3. For any bigraded solid harmonic $P$ of total degree $m$, the operator $T_{P}$ defined above is bounded on $L^{p}\left(\mathbb{C}^{n}\right), 1<p<\infty$, and is of weak type $(1,1)$.

We now consider the Riesz transforms $G_{\lambda}(P) H(\lambda)^{-\frac{m}{2}}$ as multipliers for the (group) Fourier transform on the Heisenberg group $\mathbb{H}^{n}$ and explain why they are natural. Recall that a Fourier multiplier for the Heisenberg group is a family of bounded linear operators $M(\lambda)$ on $L^{2}\left(\mathbb{R}^{n}\right)$ and the multiplier transformation $T_{M}$ is defined by $\pi_{\lambda}\left(T_{M} f\right)=\hat{f}(\lambda) M(\lambda)$ where $\hat{f}(\lambda)=\pi_{\lambda}(f)$ is the group Fourier transform on the Heisenberg group. Such operators are precisely those which are given by convolution with certain kernels on $\mathbb{H}^{n}$ and hence commute with translations. The unitary group $U(n)$ acts on $\mathbb{H}^{n}$ by automorphisms which leads to an action on $L^{2}\left(\mathbb{H}^{n}\right)$. 
Let $\mathcal{H}_{p, q}$ stand for the space of bigraded solid harmonics of bidegree $(p, q)$, which supports an irreducible unitary representation $R(\sigma)$ of $U(n)$. In what follows we let $P_{k}(\lambda)$ stand for the orthogonal projection of $L^{2}\left(\mathbb{R}^{n}\right)$ onto the $k$-th eigenspace of $H(\lambda)$. We also denote by $\rho(\sigma) f$ the action of $\sigma \in U(n)$ on functions defined on $\mathbb{H}^{n}$, i.e., $\rho(\sigma) f(z, t)=f\left(\sigma^{-1} z, t\right)$ and by $\delta_{r}, r>0$ the nonisotropic dilation $\delta_{r} f(z, t)=f\left(r z, r^{2} t\right)$.

Theorem 1.4. Let $T$ be a translation invariant operator taking $L^{2}\left(\mathbb{H}^{n}\right)$ into $L^{2}\left(\mathbb{H}^{n}, \mathcal{H}_{p, q}\right)$ and let $M(\lambda)$ be the corresponding Fourier multiplier. Assume that

(i) $R(\sigma) T f(z, t)=\rho(\sigma) T \rho\left(\sigma^{*}\right) f(z, t)$ for every $\sigma \in U(n)$,

(ii) $T \delta_{r} f(z, t)=\delta_{r} T f(z, t)$ for every $r>0$, and

(iii) $M(\lambda) P_{k}(\lambda)=((2 k+n)|\lambda|)^{-\frac{1}{2}(p+q)} S(\lambda)$ for some (unbounded) operator $S(\lambda)$.

Then for any linear functional $\beta$ of $\mathcal{H}_{p, q}$ the operator $\beta(T) f=\beta(T f)$ is a linear combination of the Riesz transforms with multipliers $G_{\lambda}(P) H(\lambda)^{-\frac{p+q}{2}}$ as $P$ runs through an orthonormal basis of $\mathcal{H}_{p, q}$.

The above theorem is the analogue of a result of Stein for higher order Riesz transforms on $\mathbb{R}^{n}$, see page 79 in [17]. For variations on the same theme we refer to the recent works [9] and [10] of Kobayashi and Nilsson. The operators on $L^{2}\left(\mathbb{H}^{n}\right)$ with multipliers $G_{\lambda}(P) H(\lambda)^{-\frac{p+q}{2}}$ turn out to be singular integral operators. Hence we get:

Theorem 1.5. Let $T$ satisfy the assumptions of the previous theorem and let $\beta$ be a linear functional on $\mathcal{H}_{p, q}$. Then $\beta(T)$ is bounded on $L^{p}\left(\mathbb{H}^{n}\right), 1<p<\infty$, and is of weak type $(1,1)$.

Given $P \in \mathcal{H}_{p, q}$ let $R_{P}$ stand for the (higher order) Riesz transform with multiplier $G_{\lambda}(P) H(\lambda)^{-\frac{p+q}{2}}$. The boundedness of these higher order Riesz transforms on $L^{p}\left(\mathbb{H}^{n}\right)$ is well known, see e.g. the works of Folland [5], Lohoué and Varopoulos [11] and Ter Elst et al. [4]. However, dimension-free estimates are not known and we conjecture that such estimates are true. Here we prove such estimates under some assumptions on $P$. Indeed, we let $P_{0}(z)=z_{j}^{p} \overline{z_{k}}, j \neq k$, and denote by $O\left(P_{0}\right)$ the orbit of $P_{0}$ under the action of $U(n)$. We then have:

Theorem 1.6. For every $P \in O\left(P_{0}\right)$, the Riesz transform $R_{P}$ satisfies the estimate $\left\|R_{P} f\right\|_{p} \leq C_{p}\|f\|_{p}$ on $L^{p}\left(\mathbb{H}^{n}\right), 1<p<\infty$, where $C_{p}$ is independent of the dimension $n$ and $P \in O\left(P_{0}\right)$.

We actually show that for any solid harmonic $P$ and $\sigma \in U(n)$ the operators $R_{P}$ and $R_{\rho(\sigma) P}$ have the same norm on $L^{p}\left(\mathbb{H}^{n}\right)$. The theorem is then proved by showing that $R_{P_{0}}$ satisfies dimension-free bounds on $L^{p}\left(\mathbb{H}^{n}\right)$. We prove this using known estimates for first order Riesz transforms and a dimension-free estimate for a particular singular integral operator on the Heisenberg group, see Section 3.

A slight strengthening of the above theorem is possible. (We are thankful to the referee for pointing this out.) Given a solid harmonic $P$ on $\mathbb{C}^{n}$ we can also consider it as a solid harmonic on $\mathbb{C}^{m}$ for any $m \geq n$ which depends only on the 
first $n$ variables. Hence we can define Riesz transforms $R_{P}^{m}$ acting on $L^{p}\left(\mathbb{H}^{m}\right)$. As $U(n)$ can be considered as a subgroup of $U(m)$ in the obvious way, the operator norm of $R_{P}^{m}$ on $L^{p}\left(\mathbb{H}^{m}\right)$ is the same as that of $R_{P_{0}}^{m}$ which is independent of $m$. Thus $R_{P}^{m}: L^{p}\left(\mathbb{H}^{m}\right) \rightarrow L^{p}\left(\mathbb{H}^{m}\right)$ satisfies estimates which are independent of $m \geq n \geq 2$ and $P \in O\left(P_{0}\right)$.

By considering functions on $\mathbb{C}^{n}$ which are homogeneous we show that the Riesz transforms $T_{z_{j}}$ and $T_{\bar{z}_{j}}$ are related to Riesz transforms for multiple Laguerre expansions studied by Nowak and Stempak [14]. We prove that the boundedness of these Laguerre-Riesz transforms are equivalent to certain weighted norm inequalities for $T_{z_{j}}$ and $T_{\overline{z_{j}}}$ on $L^{p}\left(\mathbb{C}^{n}\right)$.

\section{Riesz transforms on the reduced Heisenberg group}

In this section we prove Theorem 1.1. As we mentioned earlier, this will be done by using a transference theorem which we now describe. Let the special Hermite operators $L_{\lambda}$ be defined by the equation $\mathcal{L}\left(e^{i \lambda t} f(z)\right)=e^{i \lambda t} L_{\lambda} f(z)$. Then it is known that

$$
L_{\lambda}=-\Delta+\lambda^{2}|z|^{2}+i \lambda N
$$

where $\Delta$ is the Laplacian on $\mathbb{C}^{n}$ and

$$
N=\sum_{j=1}^{n}\left(x_{i} \frac{\partial}{\partial y_{j}}-y_{i} \frac{\partial}{\partial x_{j}}\right)
$$

We can also write $L_{\lambda}$ as

$$
L_{\lambda}=-\frac{1}{2} \sum_{j=1}^{n}\left(Z_{j}(\lambda) \bar{Z}_{j}(\lambda)+\bar{Z}_{j}(\lambda) Z_{j}(\lambda)\right),
$$

where $Z_{j}\left(e^{i \lambda t} f(z)\right)=e^{i \lambda t} Z_{j}(\lambda) f(z)$ and $\bar{Z}_{j}\left(e^{i \lambda t} f(z)\right)=e^{i \lambda t} \bar{Z}_{j}(\lambda) f(z)$. In view of this, the Riesz transforms on the Heisenberg group can be written as

$$
R_{j} f(z, t)=\frac{1}{2 \pi} \int_{-\infty}^{\infty} e^{-i \lambda t} R_{j}(\lambda) f^{\lambda}(z) d \lambda,
$$

where $R_{j}(\lambda)=Z_{j}(\lambda) L_{\lambda}^{-1 / 2}$. We have a similar expression for $\bar{R}_{j}$.

Therefore, we can view the Riesz transforms as operator valued multipliers for the (Euclidean) Fourier transform on $\mathbb{R}$. Indeed, if we let $X=L^{p}\left(\mathbb{C}^{n}\right)$ then $L^{p}\left(\mathbb{H}^{n}\right)$ can be identified with $L^{p}(\mathbb{R}, X)$ and $R_{j}$ are multiplier transforms corresponding to the (operator valued) multipliers $R_{j}(\lambda)$. Using the same notation for Riesz transforms on $\mathbb{H}^{n} / \Gamma$, they are given by

$$
R_{j} f(z, t)=\frac{1}{2 \pi} \sum_{k=-\infty}^{\infty} e^{-i k t} R_{j}(k) f^{k}(z) .
$$


Thus the Riesz transforms for $\mathbb{H}^{n} / \Gamma$ are multipliers for the Fourier series acting on $L^{p}\left(S^{1}, X\right)$. In order to treat the vector of the Riesz transforms we set

$$
\mathcal{R} f=\left(R_{1} f, R_{2} f, \ldots, R_{n} f, \bar{R}_{1} f, \bar{R}_{2} f, \ldots, \bar{R}_{n} f\right)
$$

and introduce the Banach space $Y=L^{p}\left(\mathbb{C}^{n}\right) \times L^{p}\left(\mathbb{C}^{n}\right) \times \cdots \times L^{p}\left(\mathbb{C}^{n}\right)(2 n$ times $)$ equipped with the norm

$$
\|f\|_{Y}=\left\|\left(\sum_{j=1}^{2 n}\left|f_{j}\right|^{2}\right)^{1 / 2}\right\|_{p}, \quad f=\left(f_{1}, \ldots, f_{2 n}\right) .
$$

Then the result of [3] can be written as

$$
\|\mathcal{R} f\|_{L^{p}(\mathbb{R}, Y)} \leq C\|f\|_{L^{p}(\mathbb{R}, X)},
$$

where $C$ is independent of the dimension. We are interested in showing that a similar estimate holds when $L^{p}(\mathbb{R}, X)$ and $L^{p}(\mathbb{R}, Y)$ are replaced by $L^{p}\left(S^{1}, X\right)$ and $L^{p}\left(S^{1}, Y\right)$ respectively. All we need in order to prove this is the following transference result.

Denote by $\left(L^{p}(\mathbb{R}, X), L^{p}(\mathbb{R}, Y)\right)$ the set of all Fourier multipliers taking $L^{p}(\mathbb{R}, X)$ into $L^{p}(\mathbb{R}, Y)$. Let $T \in\left(L^{p}(\mathbb{R}, X), L^{p}(\mathbb{R}, Y)\right)$ and let $m$ be the corresponding multiplier so that

$$
T f(t)=\frac{1}{2 \pi} \int_{-\infty}^{\infty} e^{-i \lambda t} m(\lambda) \hat{f}(\lambda) d \lambda .
$$

We can define a periodised operator $\tilde{T}$ by

$$
\tilde{T} f(t)=\frac{1}{2 \pi} \sum_{k=-\infty}^{\infty} e^{-i k t} m(k) \hat{f}(k)
$$

for every $f \in L^{p}\left(S^{1}, X\right)$.

Theorem 2.1. Suppose $1 \leq p \leq \infty$ and $T \in\left(L^{p}(\mathbb{R}, X), L^{p}(\mathbb{R}, Y)\right)$, where $X$ and $Y$ are Banach spaces. Let $m$ be the multiplier corresponding to $T$ and assume that $m$ is continuous at every point of $\mathbb{Z}$, the set of all integers. Then the periodised operator $\tilde{T}$ belongs to $\left(L^{p}\left(S^{1}, X\right), L^{p}\left(S^{1}, Y\right)\right)$ and satisfies $\|\tilde{T}\| \leq\|T\|$.

When $X=Y=\mathbb{C}$, this theorem is due to De Leeuw, and a proof can be found in Stein-Weiss [19], see Theorem 3.8, Chapter VII. The proof given there can be easily modified to yield the above version of transference. We leave the details to the interested reader. Once we have the above theorem it is clear that Theorem 1.1 follows from the corresponding result for Riesz transforms on $\mathbb{H}^{n}$ proved in [3]. We can also treat higher order Riesz transforms: for every $P \in \mathcal{H}_{p, q}$ let us define

$$
\tilde{R}_{P} f(z, t)=\frac{1}{2 \pi} \sum_{k=-\infty}^{\infty} e^{-i k t} T_{P} f^{k}(z),
$$

where $T_{P}$ is as in Theorem 1.3. Then:

Theorem 2.2. The operators $\tilde{R}_{P}$ defined above are all bounded on $L^{p}\left(\mathbb{H}^{n} / \Gamma\right)$ for $1<p<\infty$. 
The first order Riesz transforms satisfy dimension-free bounds and we expect the same for higher order Riesz transforms. This is an interesting open problem even in the case of $\mathbb{H}^{n}$. Our partial result, Theorem 1.6, naturally has an analogue for $\mathbb{H}^{n} / \Gamma$.

\section{Higher order Riesz transforms on the Heisenberg group}

In this section we prove Theorems 1.4, 1.5, and 1.6 stated in the introduction. The statement and the proof of Theorem 1.4 are both inspired by the corresponding result of Stein for the Euclidean case stated in [17] (see Chapter III, Section 4.8, page 79$)$. For the proof we need some facts about Weyl transform $W_{\lambda}$ and Weyl correspondence $G_{\lambda}$. We closely follow the notations from the monograph [21], to which we refer for any unexplained terminology. For each non-zero real $\lambda$, the Weyl transform $W_{\lambda}(f)$ of a function on $\mathbb{C}^{n}$ is defined as the operator

$$
W_{\lambda}(f)=\int_{\mathbb{C}^{n}} f(z) \pi_{\lambda}(z, 0) d z
$$

where $\pi_{\lambda}$ is the Schrödinger representation of $\mathbb{H}^{n}$ with parameter $\lambda$. The Weyl correspondence is then defined by $G_{\lambda}(f)=W_{\lambda}\left(\mathcal{F}_{\lambda}(f)\right)$ where $\mathcal{F}_{\lambda}(f)$ is the symplectic Fourier transform of $f$. As in the introduction we denote by $\mathcal{H}_{p, q}$ the space of bigraded spherical harmonics of bidegree $(p, q)$.

We now recall the Hecke-Bochner formula for the Weyl transform proved by Geller [6]. We remark that the notation we use here is different from that of Geller as we follow [22]. When $f$ is radial and $P$ is a bigraded solid harmonic of bidegree $(p, q)$ we have $($ for $\lambda>0)$

$$
W_{\lambda}(P f)=G_{\lambda}(P)\left(\sum_{k=p}^{\infty} R_{k-p}^{\lambda}(f) P_{k}(\lambda)\right),
$$

where $P_{k}(\lambda)$ are the projections associated to the Hermite operator $H(\lambda)$ and

$$
R_{k}^{\lambda}(f)=\frac{\Gamma(k-p+1) \Gamma(n)}{\Gamma(k+q+n)} \int_{\mathbb{C}^{n+p+q}} f(|z|) \varphi_{k, \lambda}^{n+p+q-1}(z) d z .
$$

In the above formula,

$$
\varphi_{k, \lambda}^{n+p+q-1}(z)=L_{k}^{n+p+q-1}\left(\frac{1}{2} \lambda|z|^{2}\right) e^{-\frac{1}{4} \lambda|z|^{2}}
$$

are the Laguerre functions of type $(n+p+q-1)$. We refer to [23] (Theorem 2.6.2) and [6] (Theorem 4.2) for more details.

We now begin with a proof of Theorem 1.4. Since $T$ is a translation invariant operator taking $L^{2}\left(\mathbb{H}^{n}\right)$ into $L^{2}\left(\mathbb{H}^{n}, \mathcal{H}_{p, q}\right)$ it is a convolution operator with a distributional kernel $k(z, t)$ taking values in $\mathcal{H}_{p, q}: T f(z, t)=f * k(z, t)$. 
Let $\left\{Y_{j}: j=1,2, \ldots, d(p, q)\right\}$ be an orthonormal basis of $\mathcal{H}_{p, q}$ consisting of spherical harmonics so that

$$
k(z, t)=\sum_{j=1}^{d(p, q)} k_{j}(z, t) Y_{j}
$$

where $k_{j}(z, t)$ are scalar valued distributions on $\mathbb{H}^{n}$. Then, we can write

$$
T f(z, t)=\sum_{j=1}^{d(p, q)} T_{j} f(z, t) Y_{j}=\sum_{j=1}^{d(p, q)} f * k_{j}(z, t) Y_{j} .
$$

The hypothesis (i), namely $\rho(\sigma) T \rho\left(\sigma^{-1}\right) f=R(\sigma) T f$, translates into

$$
R(\sigma) T f(z, t)=T \rho\left(\sigma^{-1}\right) f\left(\sigma^{-1} z, t\right)=\sum_{j=1}^{d(p, q)} T_{j} \rho\left(\sigma^{-1}\right) f\left(\sigma^{-1} z, t\right) Y_{j} .
$$

Let $\left(a_{i, j}(\sigma)\right)$ stand for the matrix corresponding to $R(\sigma)$ in the basis $\left\{Y_{j}: j=\right.$ $1,2, \ldots, d(p, q)\}$. Then we have

$$
\sum_{j=1}^{d(p, q)} a_{i, j}(\sigma) T_{j} f(z, t)=T_{i} \rho\left(\sigma^{-1}\right) f\left(\sigma^{-1} z, t\right),
$$

which gives after a simple calculation the relation

$$
f * \sum_{j=1}^{d(p, q)} a_{i, j}(\sigma) k_{j}(z, t)=f * \rho\left(\sigma^{-1}\right) k_{i}(z, t)
$$

This shows that

$$
R(\sigma) k(z, t)=\rho\left(\sigma^{-1}\right) k(z, t)=k(\sigma z, t), \quad \sigma \in U(n) .
$$

From this relation we observe that for any unit vector $w \in \mathbb{C}^{n}, k(w, t)$ as an element of $\mathcal{H}_{p, q}$ is invariant under all $\sigma \in U(n)$ which fixes $w$. Therefore, there is a scalar valued function $c(w, t)$ such that $k(w, t)=c(w, t) Y_{w}$, where $Y_{w}$ is the zonal harmonic with pole at $w$.

For any two unit vectors $z$ and $w$ we can always find $\sigma \in U(n)$ such that $\sigma z=w$. Hence $R(\sigma) k(z, t)=k(\sigma z, t)=k(w, t)$ leads to the equation

$$
c(w, t) Y_{w}=R(\sigma) k(z, t)=c(z, t) R(\sigma) Y_{z}
$$

Evaluating both sides at $w$ and noting that $R(\sigma) Y_{z}(w)=Y_{z}(z)=Y_{w}(w)$, we see that $c(z, t)$ is a constant as long as $|z|=1$ and hence $k(z, t)=c(t) Y_{z}$ for all $z \in S^{2 n-1}$. Now the hypothesis $\delta_{r} T=T \delta_{r}$ translates into the homogeneity $k\left(r z, r^{2} t\right)=r^{-2 n-2} k(z, t)$. Therefore, for any $z \in \mathbb{C}^{n}$,

$$
k(z, t)=|z|^{-2 n-2} k\left(\frac{z}{|z|}, \frac{t}{|z|^{2}}\right)=|z|^{-2 n-2} c\left(\frac{t}{|z|^{2}}\right) Y_{\frac{z}{|z|}} .
$$


Let $P_{j}$ stand for the solid harmonic satisfying $P_{j}(z)=|z|^{p+q} Y_{j}(z /|z|)$. Expanding $k(z, t)$ in terms of $Y_{j}$ and noting that $\left(Y_{z /|z|}, Y_{j}\right)_{\mathcal{H}_{p, q}}=Y_{j}(z /|z|)=|z|^{-p-q} P_{j}(z)$, we get

$$
k_{j}(z, t)=|z|^{-2 n-p-q-2} c\left(\frac{t}{|z|^{2}}\right) P_{j}(z)=g_{j}(z, t) P_{j}(z) .
$$

Note that $g_{j}(z, t)$ is a radial function satisfying the homogeneity condition

$$
\delta_{r} g_{j}=r^{-2 n-p-q-2} g_{j}
$$

Let $\beta$ be any linear functional on $\mathcal{H}_{p, q}$. Then we have

$$
\beta(T f)=\sum_{j=1}^{d(p, q)} c_{j} T_{j} f=\sum_{j=1}^{d(p, q)} c_{j} f * k_{j}
$$

The operator $T_{j}$ is a Fourier multiplier operator with multiplier $M_{j}(\lambda)=W_{\lambda}\left(k_{j}^{\lambda}\right)$, which can be calculated using Hecke-Bochner formula. As $k_{j}^{\lambda}(z)=g_{j}^{\lambda}(z) P_{j}(z)$ it follows that for $\lambda>0$

$$
W_{\lambda}\left(P_{j} g_{j}^{\lambda}\right)=G_{\lambda}\left(P_{j}\right)\left(\sum_{k=p}^{\infty} R_{k-p}^{\lambda}\left(g_{j}^{\lambda}\right) P_{k}(\lambda)\right),
$$

where

$$
R_{k}^{\lambda}\left(g_{j}^{\lambda}\right)=\frac{\Gamma(k-p+1) \Gamma(n)}{\Gamma(k+q+n)} \int_{\mathbb{C}^{n+p+q}} g_{j}^{\lambda}(z) \varphi_{k, \lambda}^{n+p+q-1}(z) d z .
$$

As $g_{j}^{\lambda}(r z)=r^{-2 n-p-q} g_{j}^{\lambda r^{2}}(z)$ we have $R_{k}^{\lambda r^{2}}\left(g_{j}^{\lambda r^{2}}\right)=r^{-p-q} R_{k}^{\lambda}\left(g_{j}^{\lambda}\right)$ and hence $R_{k}^{\lambda}\left(g_{j}^{\lambda}\right)=R_{k}^{1}\left(g_{j}^{1}\right) \lambda^{-(p+q) / 2}$.

Therefore, we have shown that the multiplier $M_{j}(\lambda)$ is of the form

$$
M_{j}(\lambda)=G_{\lambda}\left(P_{j}\right)\left(\sum_{k=p}^{\infty} c_{k-p} P_{k}(\lambda)\right) \lambda^{-(p+q) / 2} .
$$

Finally, the hypothesis on $M_{j}(\lambda) P_{k}(\lambda)$ shows that $c_{k-p}=(2 k+n)^{-(p+q) / 2}$ for all $k \geq p$. It can be checked that $G_{\lambda}\left(P_{j}\right) P_{k}(\lambda)=0$ for $k<p$ and consequently $M_{j}(\lambda)=G_{\lambda}\left(P_{j}\right) H(\lambda)^{-(p+q) / 2}$, which proves Theorem 1.4.

We now turn our attention to the proofs of Theorems 1.5 and 1.6. Recall that for $P \in \mathcal{H}_{p, q}$ we have defined $R_{P}$ as the (higher order) Riesz transform with multiplier $G_{\lambda}(P) H(\lambda)^{-\frac{p+q}{2}}$. As defined earlier, $O\left(P_{0}\right)$ stands for the orbit of $P_{0}$ under the action of $U(n)$. In other words, $O\left(P_{0}\right)$ is the set of all $\rho(\sigma) P_{0}$ as $\sigma$ ranges over $U(n)$.

Proposition 3.1. In order to prove dimension-free estimate for $R_{P}, P \in O\left(P_{0}\right)$, it is enough to consider $P_{0}(z)=z_{j}^{p} \bar{z}_{k}^{q}$. (Here we assume $n \geq 2$ ). 
Proof. To prove the proposition we make use of the operators $\rho(\sigma)$ introduced earlier. For $f \in L^{p}\left(\mathbb{H}^{n}\right),\|\rho(\sigma) f\|_{p}=\|f\|_{p}$, and hence we need to show that

$$
\int_{\mathbb{H}^{n}}\left|\rho(\sigma)\left(R_{P} \rho\left(\sigma^{*}\right) f\right)(z, t)\right|^{p} d z d t \leq C_{p} \int_{\mathbb{H}^{n}}|f(z, t)|^{p} d z d t .
$$

From the theorem of Stone-von Neumann we know that, for every $\sigma \in U(n)$,

$$
\pi_{\lambda}(\sigma . z, t)=\mu_{\lambda}(\sigma)^{*} \pi_{\lambda}(z, t) \mu_{\lambda}(\sigma)
$$

where $\mu_{\lambda}(\sigma)$ are certain unitary operators on $L^{2}\left(\mathbb{R}^{n}\right)$. In view of this, a simple calculation shows that

$$
\pi_{\lambda}(\rho(\sigma) f)=\mu_{\lambda}(\sigma) \hat{f}(\lambda) \mu_{\lambda}(\sigma)^{*}
$$

and hence

$$
\pi_{\lambda}\left(\rho(\sigma) R_{P} \rho\left(\sigma^{*}\right) f\right)=\hat{f}(\lambda) \mu_{\lambda}(\sigma) G_{\lambda}(P) \mu_{\lambda}(\sigma)^{*} H(\lambda)^{-\frac{p+q}{2}},
$$

where we have made use of the fact that $\mu_{\lambda}(\sigma)$ commutes with $H(\lambda)$. However,

$$
\mu_{\lambda}(\sigma) G_{\lambda}(P) \mu_{\lambda}(\sigma)^{*}=G_{\lambda}(\rho(\sigma) P),
$$

and the proposition is proved by choosing $\sigma$ such that $\rho(\sigma) P(z)=z_{j}^{p} \bar{z}_{k}^{q}$, which is possible for any $P \in O\left(P_{0}\right)$.

The proof of the above proposition actually shows that if $R_{P}$ is bounded on $L^{p}\left(\mathbb{H}^{n}\right)$ then so is $R_{(\rho(\sigma) P)}$. As $R$ is a unitary representation of $U(n)$ on $\mathcal{H}_{p, q}$, any $P \in \mathcal{H}_{p, q}$ is a finite linear combination of elements from the orbit of $P_{0}(z)$. This proves Theorem 1.5.

We continue with our proof of Theorem 1.6. As $j \neq k$ are arbitrary, we can very well assume that $P_{0}(z)=z_{1}^{p} \bar{z}_{2}^{q}$. The operator $G_{\lambda}(P)$ associated to it by the Weyl correspondence is explicitly known and given by $A_{2}(\lambda)^{q} A_{1}(\lambda)^{* p}$, see Geller [6] or [23]. Thus it is enough to consider the Riesz transform with this multiplier. Without loss of generality we can assume that $q \geq p$. Since $A_{2}(\lambda) A_{1}(\lambda)^{*}$ commutes with $H(\lambda)$ (as can be easily checked by testing against the Hermite functions $\Phi_{\alpha}^{\lambda}$ ) the multiplier can be written as

$$
A_{2}(\lambda)^{q} A_{1}(\lambda)^{* p} H(\lambda)^{-\frac{p+q}{2}}=A_{2}(\lambda)^{q-p} H(\lambda)^{-\frac{q-p}{2}} A_{2}(\lambda)^{p} A_{1}(\lambda)^{* p} H(\lambda)^{-p} .
$$

Furthermore, using the commutativity again, we have

$$
A_{2}(\lambda)^{p} A_{1}(\lambda)^{* p} H(\lambda)^{-p}=\left(A_{2}(\lambda) A_{1}(\lambda)^{*} H(\lambda)^{-1}\right)^{p},
$$

which can be put in the form

$$
\left(H(\lambda)^{-1 / 2} A_{2}(\lambda) A_{1}(\lambda)^{*} H(\lambda)^{-1 / 2}\right)^{p}
$$

It is now clear that the operator with multiplier $A_{2}(\lambda)^{p} A_{1}(\lambda)^{* p} H(\lambda)^{-p}$ satisfies a dimension-free estimate, since the first order Riesz transforms with multipliers $H(\lambda)^{-1 / 2} A_{2}(\lambda)$ and $A_{1}(\lambda)^{*} H(\lambda)^{-1 / 2}$ satisfy such estimates. Here we have made 
use of the fact that the multiplier $H(\lambda)^{-1 / 2} A_{2}(\lambda)$ corresponds to the adjoint of the Riesz transform whose multiplier is $A_{2}(\lambda)^{*} H(\lambda)^{-1 / 2}$. Therefore, Theorem 1.6 will be proved once we have:

Theorem 3.2. For any integer $m \geq 1$, the Riesz transform with multiplier $A_{2}(\lambda)^{m}$ $H(\lambda)^{-\frac{m}{2}}$ satisfies dimension-free estimates.

Proof. Writing

$$
A_{2}(\lambda)^{m} H(\lambda)^{-m / 2}=\prod_{j=0}^{m-1} H(\lambda)^{j / 2} A_{2}(\lambda) H(\lambda)^{-(j+1) / 2},
$$

we only need to treat multipliers of the form

$$
H(\lambda)^{j / 2} A_{2}(\lambda) H(\lambda)^{-(j+1) / 2}
$$

for $j \geq 1$, which we do using an induction argument. Recalling that

$$
H(\lambda)=\frac{1}{2} \sum_{j=1}^{n}\left(A_{j}(\lambda) A_{j}^{*}(\lambda)+A_{j}^{*}(\lambda) A_{j}(\lambda)\right)
$$

and the commutation relation

$$
A_{j}(\lambda) A_{j}(\lambda)^{*}-A_{j}^{*}(\lambda) A_{j}(\lambda)=-4 \lambda I
$$

we can show that

$$
H(\lambda) A_{j}(\lambda)=A_{j}(\lambda) H(\lambda)+4 \lambda A_{j}(\lambda)
$$

In view of this,

$$
H(\lambda)^{j / 2} A_{2}(\lambda) H(\lambda)^{-(j+1) / 2}=H(\lambda)^{(j-2) / 2} H(\lambda) A_{2}(\lambda) H(\lambda)^{-(j+1) / 2}
$$

reduces to

$$
H(\lambda)^{(j-2) / 2} A_{2}(\lambda) H(\lambda)^{-(j-1) / 2}+4 H(\lambda)^{(j-2) / 2} A_{2}(\lambda) H(\lambda)^{-(j-1) / 2} \lambda H(\lambda)^{-1} .
$$

If we can show that the operator with multiplier $\lambda H(\lambda)^{-1}$ satisfies dimension-free estimates then we can use an induction on $j$ to complete the proof of the above theorem. Thus the proof is completed by proving the following result.

Theorem 3.3. The multiplier $\lambda H(\lambda)^{-1}$ defines a singular integral operator on $\mathbb{H}^{n}$ which satisfies dimension-free bounds on all $L^{p}$ spaces, $1<p<\infty$.

Proof. The boundedness of this operator is well known; see Folland [5] and Stein [18]. Indeed, the above multiplier corresponds to $T \mathcal{L}^{-1}$, where $T=\frac{\partial}{\partial t}$, and is given by convolution with the kernel $K(z, t)=T \varphi_{0}(z, t)$, where

$$
\varphi_{0}(z, t)=c_{n}\left(|z|^{4}+t^{2}\right)^{-n / 2}
$$


is the fundamental solution for the sublaplacian found by Folland [5]. Here the constant $c_{n}$ is given by

$$
c_{n}^{-1}=n(n+2) \int_{\mathbb{H}^{n}}\left(1+t^{2}+|z|^{4}\right)^{-(n+4) / 2}|z|^{2} d z d t .
$$

It is clear that $K(z, t)$ is an odd kernel which is homogeneous of degree $(-2 n-2)$, and hence defines a bounded operator on $L^{p}\left(\mathbb{H}^{n}\right), 1<p<\infty$. The norm of this operator can be estimated using a result of Christ [2] as presented in Strichartz [20] (Lemma 3.1). This shows that the norm on $L^{p}$ is bounded by

$$
C_{p} \int_{\mathbb{C}^{n}}|K(z, 1)| d z
$$

where $C_{p}$ depends only on $p$. Thus we are left with proving the inequality

$$
n c_{n} \int_{\mathbb{C}^{n}}\left(1+|z|^{4}\right)^{-n / 2-1} d z \leq C
$$

for some constant $C$ independent of $n$. This follows from the next lemma.

Lemma 3.4. There is a constant $C$ independent of $n$ such that

$$
\frac{\int_{\mathbb{C}^{n}}\left(1+|z|^{4}\right)^{-n / 2-1} d z}{(n+2) \int_{\mathbb{H}^{n}}\left(1+t^{2}+|z|^{4}\right)^{-(n+4) / 2}|z|^{2} d z d t} \leq C
$$

for all values on $n$.

Proof. Integrating in polar coordinates, the above ratio reduces to

$$
\frac{\int_{0}^{\infty}\left(1+r^{4}\right)^{-n / 2-1} r^{2 n-1} d r}{2(n+2) \int_{0}^{\infty} \int_{0}^{\infty}\left(1+t^{2}+r^{4}\right)^{-(n+4) / 2} r^{2 n+1} d r d t} .
$$

By a simple change of variables the integral on the numerator can be seen to be the beta integral

$$
\frac{1}{4} \int_{0}^{\infty}(1+t)^{-n / 2-1} t^{n / 2-1} d t=\frac{\Gamma(1) \Gamma(n / 2)}{4 \Gamma(n / 2+1)} .
$$

Similarly, the integral in the denominator becomes the product

$$
\left(\int_{0}^{\infty}\left(1+t^{2}\right)^{-3 / 2} d t\right)\left(\int_{0}^{\infty}\left(1+u^{4}\right)^{-(n+4) / 2} u^{(2 n+1)} d u\right) .
$$

As before,

$$
\int_{0}^{\infty}\left(1+t^{2}\right)^{-3 / 2} d t=\frac{\Gamma(1 / 2) \Gamma(1)}{2 \Gamma(3 / 2)}
$$

and

$$
\int_{0}^{\infty}\left(1+u^{4}\right)^{-(n+4) / 2} u^{(2 n+1)} d u=\frac{\Gamma(3 / 2) \Gamma((n+1) / 2)}{4 \Gamma((n+4) / 2)} .
$$


Thus the ratio of the two integrals is given by

$$
\frac{\Gamma(n / 2)}{4 \Gamma(n / 2+1)} \frac{8 \Gamma(n / 2+2)}{\Gamma(1 / 2) \Gamma((n+1) / 2)} .
$$

Simplifying this we see that the ratio in the lemma is given by

$$
\pi^{-1 / 2} \frac{\Gamma(n / 2)}{\Gamma((n+1) / 2)}
$$

which is clearly a bounded function of $n$.

\section{Riesz transforms for Hermite and Laguerre expansions}

In this section we consider Riesz transforms associated to Hermite and Laguerre expansions. First we can deduce Theorem 1.3 from the corresponding result for the reduced Heisenberg group. We fix $\lambda=1$ and simply write $W$ and $G$ instead of $W_{1}$ and $G_{1}$. Consider the Weyl multiplier $T_{P}$ defined by

$$
W\left(T_{P} f\right)=W(f) G(P) H^{-(p+q) / 2},
$$

where $P \in \mathcal{H}_{p, q}$. The Weyl multipliers can be considered as higher order Riesz transforms for the special Hermite operator. Recall that the special Hermite operator $L$ is defined by $\mathcal{L}\left(e^{i t} f(z)\right)=e^{i t} L f(z)$. The spectral decomposition of this operator is given by

$$
L f=(2 \pi)^{-n} \sum_{k=0}^{\infty} f \times \varphi_{k}^{n-1},
$$

where $\varphi_{k}^{n-1}(z)$ are Laguerre functions of type $(n-1)$ and $\times$ stands for the twisted convolution:

$$
f \times g(z)=\int_{\mathbb{C}^{n}} f(z-w) g(w) e^{\frac{i}{2} \Im(z \cdot \bar{w})} d w .
$$

When $P(z)=z_{j}\left(\right.$ resp. $\left.\bar{z}_{j}\right)$ it follows from the work of Geller that $G(P)=A_{j}$ (resp. $G(P)=A_{j}^{*}$ ), and hence $T_{z_{j}}=Z_{j}(1) L^{-1 / 2}$ and $\left.T_{\bar{z}_{j}}=\overline{Z_{j}}(1)\right) L^{-1 / 2}$ are the Riesz transforms for the special Hermite expansions. More generally if $P(z)=$ $\sum_{|\alpha|=p,|\beta|=q} c_{\alpha, \beta} z^{\alpha} \bar{z}^{\beta}$ then $G(P)$ is obtained by replacing $z_{j}$ and $\bar{z}_{j}$ by $A_{j}$ and $A_{j}^{*}$, respectively. Though the following result can be deduced from results on the reduced Heisenberg group by considering functions of the form $e^{i t} f(z)$ we can give a simple direct proof based on the Hecke-Bochner formula for the Weyl transform.

Theorem 4.1. For every $P \in \mathcal{H}_{p, q}$, the Riesz transforms $T_{P}$ are bounded on $L^{p}\left(\mathbb{C}^{n}\right), 1<p<\infty$, and are also weak type $(1,1)$. The first order Riesz transforms satisfy dimension-free bounds.

Proof. The proof is very similar to the one given in [21] for the cases $P(z)=z_{j}$ and $P(z)=\bar{z}_{j}$. Hence we will only give a sketch of the proof. From the Hecke-Bochner formula for the special Hermite projections (see Geller [6] and [23]) it follows that

$$
G(P) P_{k}=c_{n}(p, q) W\left(P \varphi_{k-p}^{n+p+q-1}\right)
$$


(see 2.6.22 in [23]), and hence

$$
G(P) H^{-(p+q) / 2}=c_{n}(p, q) W\left(P K_{m}\right),
$$

where $m=p+q$ and $K_{m}$ is the kernel defined by

$$
K_{m}(z)=\sum_{k=0}^{\infty}(2 k+2 p+n)^{-m / 2} \varphi_{k}^{n+p+q-1}(z) .
$$

On the other hand, using the relations

$$
W\left(Z_{j} f\right)=W(f) A_{j}, \quad W\left(\bar{Z}_{j} f\right)=W(f) A_{j}^{*}
$$

we can obtain

$$
W\left(L^{-m / 2} P(Z, \bar{Z}) f\right)=W(f) G(P) H^{-m / 2},
$$

so that a comparison with the above formula shows that

$$
L^{-m / 2} P(Z, \bar{Z}) f(z)=f \times\left(P K_{m}\right)(z) .
$$

The kernel $K_{m}$ can be expressed in terms of the heat kernel for $L$ on $\mathbb{C}^{n+p+q}$ and hence estimated. It turns out that $L^{-m / 2} P(Z, \bar{Z})$ is an oscillatory singular integral operator. Hence, by the results of Ricci-Stein [16] and Chanillo-Christ [1], it is bounded on $L^{p}\left(\mathbb{C}^{n}\right)$ for $1<p<\infty$ and of weak type $(1,1)$. By considering the adjoint we get the desired result.

We now deduce some results for Riesz transforms associated to multiple Laguerre expansions. In terms of special Hermite functions the spectral decomposition of $L$ takes the form

$$
f(z)=\sum_{\alpha, \beta}\left(f, \Phi_{\alpha, \beta}\right) \Phi_{\alpha, \beta}(z) .
$$

When $f$ is a polyradial function, i.e., $f(\sigma z)=f(z)$ for all diagonal matrices $\sigma$ in $U(n)$, the special Hermite expansion of $f$ reduces to a multiple Laguerre expansion. More generally, when $f$ is a polyradial function the special Hermite expansion of $g(z)=z^{m} f(z)$ where $m \in \mathbb{N}^{n}$ takes the form

$$
g(z)=\sum_{\alpha}\left(\int_{\mathbb{C}^{n}} g(z) \overline{\Phi_{\alpha, \alpha+m}}(w) d w\right) \Phi_{\alpha, \alpha+m}(z) .
$$

Indeed, we have the formula (see Theorem 1.3.5 in [21])

$$
\Phi_{\alpha, \alpha+m}(z)=(2 \pi)^{-n / 2}\left(\frac{\alpha !}{(\alpha+m) !}\right)^{1 / 2} 2^{-|m| / 2}(-i)^{|m|} z^{m} \prod_{j=1}^{n} \varphi_{\alpha_{j}}^{m_{j}}\left(z_{j}\right),
$$

and hence in the expansion only the term $\left(g, \Phi_{\alpha, \alpha+m}\right)$ survives. Note that

$$
\begin{aligned}
& \left(\int_{\mathbb{C}^{n}} g(z) \overline{\Phi_{\alpha, \alpha+m}}(w) d w\right) \Phi_{\alpha, \alpha+m}(z) \\
& \quad=(2 \pi)^{-n} \frac{\alpha !}{(\alpha+m) !}\left(\frac{z}{2}\right)^{m}\left(\int_{\mathbb{C}^{n}} f(w) \prod_{j=1}^{n} \varphi_{\alpha_{j}}^{m_{j}}\left(w_{j}\right)\left|w_{j}\right|^{2 m_{j}+1} d w\right) \prod_{j=1}^{n} \varphi_{\alpha_{j}}^{m_{j}}\left(z_{j}\right) .
\end{aligned}
$$


We now define multiple Laguerre functions of type $m$ by

$$
\Psi_{\alpha}^{m}(r)=\left(\frac{2^{-|m|} \alpha !}{(\alpha+m) !}\right)^{1 / 2} \prod_{j=1}^{n} \varphi_{\alpha_{j}}^{m_{j}}\left(r_{j}\right)
$$

where $r=\left(r_{j}\right), r_{j} \geq 0$. Here $\varphi_{\alpha_{j}}^{m_{j}}\left(r_{j}\right)$ stands for $\varphi_{\alpha_{j}}^{m_{j}}\left(z_{j}\right)$ with $\left|z_{j}\right|=r_{j}$. These functions form an orthonormal basis for $L^{2}\left(\mathbb{R}_{+}^{n}, d \mu_{m}\right)$, where

$$
d \mu_{m}(r)=\prod_{j=1}^{n} r_{j}^{2 m_{j}+1} d r_{j}
$$

The multiple Laguerre expansion of type $m$ of a function $f \in L^{2}\left(\mathbb{R}_{+}^{n}, d \mu_{m}\right)$ is related to the special Hermite expansion of $g(z)=z^{m} f(r), r_{j}=\left|z_{j}\right|$. Indeed,

$$
\sum_{\alpha}\left(g, \Phi_{\alpha, \alpha+m}\right) \Phi_{\alpha, \alpha+m}(z)=z^{m} \sum_{\alpha}\left(f, \Psi_{\alpha}^{m}\right) \Psi_{\alpha}^{m}(r) .
$$

Consider the Riesz transform $T_{\bar{z}_{j}}=\bar{Z}_{j}(1) L^{-1 / 2}$ applied to the function $g$. Since

$$
\bar{Z}_{j}(1) \Phi_{\alpha, \beta}=i\left(2 \alpha_{j}\right)^{1 / 2} \Phi_{\alpha-e_{j}, \beta},
$$

it follows that

$$
T_{\bar{z}_{j}} g(z)=i \sum_{\alpha}\left(2 \alpha_{j}\right)^{1 / 2}(2|\alpha|+n)^{-1 / 2}\left(g, \Phi_{\alpha, \alpha+m}\right) \Phi_{\alpha-e_{j}, \alpha+m}(z),
$$

which can be expressed in terms of $f$ as

$$
T_{\bar{z}_{j}} g(z)=i z^{m+e_{j}} \sum_{\alpha}\left(2 \alpha_{j}\right)^{1 / 2}(2|\alpha|+n)^{-1 / 2}\left(f, \Psi_{\alpha}^{m}\right) \Psi_{\alpha-e_{j}}^{m+e_{j}}(r) .
$$

Thus we have (with $r_{j}=\left|z_{j}\right|$ )

$$
T_{\bar{z}_{j}} g(z)=i z^{m} \frac{z_{j}}{\left|z_{j}\right|} R_{j, m} f(r)
$$

where

$$
R_{j, m} f(r)=r_{j} \sum_{\alpha}\left(2 \alpha_{j}\right)^{1 / 2}(2|\alpha|+n)^{-1 / 2}\left(f, \Psi_{\alpha}^{m}\right) \Psi_{\alpha-e_{j}}^{m+e_{j}}(r)
$$

are the Riesz transforms associated to Laguerre expansions of type $m$. These transforms have been studied by Nowak and Stempak [14] and [15]. From the above relation we obtain:

Theorem 4.2. The Laguerre-Riesz transforms $R_{j, m}$ are bounded on $L^{p}\left(\mathbb{R}_{+}^{n}, d \mu_{m}\right)$ if and only if the $T_{\bar{z}_{j}}$ satisfy the weighted norm inequality

$$
\int_{\mathbb{C}^{n}}\left|T_{\bar{z}_{j}} g(z)\right|^{p} \prod_{j=1}^{n}\left|z_{j}\right|^{m_{j}(2-p)} d z \leq C \int_{\mathbb{C}^{n}}|g(z)|^{p} \prod_{j=1}^{n}\left|z_{j}\right|^{m_{j}(2-p)} d z
$$

for all functions $g$ which are m-homogeneous, i.e., $g(z)=z^{m} f(z)$, where $f$ is polyradial. 
When $m=0$, we do have the boundedness of $T_{\bar{z}_{j}}$ on $L^{p}\left(\mathbb{C}^{n}\right), 1<p<\infty$, and hence the Riesz transforms $R_{j, 0}$ are all bounded on $L^{p}\left(\mathbb{R}_{+}^{n}, d \mu_{m}\right)$. For general $m$, we can use the result of [14] which says that $R_{j, m}$ are bounded on $L^{p}\left(\mathbb{R}_{+}^{n}, d \mu_{m}\right)$ to obtain the following result:

Corollary 4.3. For any $m \in \mathbb{N}^{n}$ we have the weighted norm inequality

$$
\int_{\mathbb{C}^{n}}\left|T_{\bar{z}_{j}} g(z)\right|^{p} \prod_{j=1}^{n}\left|z_{j}\right|^{m_{j}(2-p)} d z \leq C \int_{\mathbb{C}^{n}}|g(z)|^{p} \prod_{j=1}^{n}\left|z_{j}\right|^{m_{j}(2-p)} d z
$$

for all m-homogeneous $g \in L^{p}\left(\mathbb{C}^{n}\right), 1<p<\infty$.

Since the Riesz transforms $T_{\bar{z}_{j}}$ are (oscillatory) singular integral operators, it is natural to expect that they will satisfy weighted norm inequalities provided the weight comes from the $A_{p}$ class. In the above inequality, the weight function

$$
w(z)=\prod_{j=1}^{n}\left|z_{j}\right|^{m_{j}(2-p)}
$$

belongs to $A_{p}$ only if

$$
2\left(1-\frac{1}{2+m_{j}}\right)<p<2\left(1+\frac{1}{m_{j}}\right)
$$

Thus for functions with certain homogeneity we can expect weighted norm inequalities under weaker assumptions. It would be interesting to characterise all weight functions for which the Riesz transforms $T_{P}$ satisfy weighted norm inequalities. We plan to return to this problem in the future.

Interchanging the roles of $T_{\bar{z}_{j}}$ and $R_{j, m}$ and using Theorem 4.1, we obtain the following:

Corollary 4.4. For any $m \in \mathbb{N}^{n}$ we have the weighted norm inequality

$$
\int_{\mathbb{R}_{+}^{n}}\left|R_{j, m} f(r)\right|^{p} \prod_{j=1}^{n}\left|r_{j}\right|^{m_{j}(p-2)} d \mu_{m} \leq C_{p} \int_{\mathbb{R}_{+}^{n}}|f(r)|^{p} \prod_{j=1}^{n}\left|r_{j}\right|^{m_{j}(p-2)} d \mu_{m}
$$

for all $f \in L^{p}\left(\mathbb{R}_{+}^{n}\right), 1<p<\infty$, where $C_{p}$ is independent of $n$ and $m$.

It would be interesting to see if such dimension-free, type-free estimates are true for general multiple Laguerre expansions of type $m$ studied by [14], where the $m_{j}$ 's are not necessarily integers. Riesz transforms for multiple Laguerre expansions of a different kind have been studied by Gutiérrez et al. in [7]. When the $m_{j}$ 's are half integers, they have shown that the Riesz transforms satisfy dimension-free bounds.

Acknowledgment. The authors are extremely thankful to the referee for his thorough and careful reading of the manuscript and for pointing out a couple of mathematical errors and a host of typos. 


\section{References}

[1] Chanillo, S. And Christ, M.: Weak $(1,1)$ bounds for oscillatory singular integrals. Duke Math. J. 55 (1987), no. 1, 141-155.

[2] Christ, M.: Hilbert transforms along curves I. Nilpotent groups. Ann. of Math. (2) 122 (1985), no. 3, 575-596.

[3] Coulhon, T., Mueller, D. And Zienkiewicz, J.: About Riesz transforms on the Heisenberg groups. Math. Ann. 305 (1996), no. 2, 369-379.

[4] ter Elst, A.F. M., Robinson, D. W. And Sikora, A.: Heat kernels and Riesz transforms on nilpotent Lie groups. Colloq. Math. 74 (1997), no. 2, 191-218.

[5] Folland, G. B.: A fundamental solution for a subelliptic operator. Bull. Amer. Math. Soc. 79 (1973), 373-376.

[6] Geller, D.: Spherical harmonics, the Weyl transform and the Fourier transform on the Heisenberg group. Canad. J. Math. 36 (1984), no. 4, 615-684.

[7] Gutiérrez, C., Incognito, A. and Torrea, J. L.: Riesz transforms, $g$-functions and multipliers for the Laguerre semigroup. Houston J. Math. 27 (2001), no. 3, 579-592.

[8] Harboure, E., De Rosa, L., Segovia, C. And Torrea, J. L.: $L^{p}$-dimension free boundedness for Riesz transforms associated to Hermite functions. Math. Ann. 328 (2004), no. 4, 653-682.

[9] Kobayashi, T. And Nilsson, A.: Indefinite higher Riesz transforms. Ark. Math. 47 (2009), no. 2, 331-344.

[10] Kobayashi, T. And Nilsson, A.: Group invariance and $L^{p}$-bounded operators. Math. Z. 260 (2008), no. 2, 335-354.

[11] Lohoué, N. and Varopoulos, N. T.: Remarques sur les transformees de Riesz sur les groupes de Lie nilpotents. C. R. Acad. Sci. Paris Ser. I Math. 301 (1985), no. 11, $559-560$.

[12] F. Lust-Piquard: Dimension free estimates for Riesz transforms associated to the harmonic oscillator on $\mathbb{R}^{d}$. Potential Anal. 24 (2006), no. 1, 47-62.

[13] Mauceri, G.: The Weyl transform and bounded operators on $L^{p}\left(\mathbb{R}^{n}\right)$. J. Funct. Anal. 39 (1980), no. 3, 408-429.

[14] Nowak, A. and Stempak, K.: Riesz transforms for multi-dimensional Laguerre function expansions. Adv. Math. 215 (2007), no. 2, 642-678.

[15] Nowak, A. And Stempak, K.: Riesz transforms and conjugacy for Laguerre expansions of Hermite type. J. Funct Anal. 244 (2007), no. 2, 399-433.

[16] Ricci, F. AND Stein, E. M.: Harmonic analysis on nilpotent groups and singular integrals. I. Oscillatory integrals. J. Funct. Anal. 73 (1987), no. 1, 179-194.

[17] Stein, E. M.: Singular integrals and differentiability properties of functions. Princeton Mathematical Series 30, Princeton University Press, Princeton, NJ, 1970.

[18] Stein, E. M.: Harmonic analysis: real variable methods, orthogonality and oscillatory integrals. Princeton Mathematical Series 43, Monographs in Harmonic Analysis III, Princeton University Press, Princeton, NJ, 1993.

[19] Stein, E. M. And Weiss, G.: Introduction to Fourier analysis on euclidean spaces. Princeton Mathematical Series 32, Princeton University Press, Princeton, NJ, 1971.

[20] Strichartz, R. S.: $L^{p}$ harmonic analysis and Radon transforms on the Heisenberg group. J. Funct. Anal. 96 (1991), no. 2, 350-406. 
[21] Thangavelu, S.: Lectures on Hermite and Laguerre expansions. Mathematical Notes 42, Princeton University Press, Princeton, NJ, 1993.

[22] Thangavelu, S.: Harmonic analysis on the Heisenberg group. Progress in Mathematics 159, Birkhäuser, Boston, MA, 1998.

[23] Thangavelu, S.: An introduction to the uncertainty principle. Hardy's theorem on Lie groups. Progress in Mathematics 217, Birkhäuser, Boston, MA, 2004.

Received December 12, 2010; revised March 7, 2011.

P. K. SAnJAY: Department of Mathematics, Indian Institute of Science, Bangalore560012 , India.

(Permanent address) Department of Mathematics, National Institute of Technology, Calicut-673 601, India.

E-mail: sanjay@math.iisc.ernet.in

Sundaram Thangavelu: Department of Mathematics, Indian Institute of Science, Bangalore-560 012, India.

E-mail: veluma@math.iisc.ernet.in

The work of the first author is supported by the All India Council for Technical Education (AICTE). The work of the second author is supported by J. C. Bose Fellowship from the Department of Science and Technology (DST) and also by a grant from UGC via DSA-SAP. 